\title{
Sieve-in-the-Middle: Improved MITM Attacks ${ }^{\star}$
}

\author{
Anne Canteaut ${ }^{1}$, María Naya-Plasencia ${ }^{1}$, and Bastien Vayssière ${ }^{2}$ \\ 1 Inria Paris-Rocquencourt, Project-Team SECRET, France \\ \{Anne.Canteaut,Maria.Naya_Plasencia\}@inria.fr, \\ 2 Université de Versailles Saint-Quentin-en-Yvelines, France \\ bastien.vayssiere.w@gmail.com
}

\begin{abstract}
This paper presents a new generic technique, named sievein-the-middle, which improves meet-in-the-middle attacks in the sense that it provides an attack on a higher number of rounds. Instead of selecting the key candidates by searching for a collision in an intermediate state which can be computed forwards and backwards, we look for the existence of valid transitions through some middle sbox. Combining this technique with short bicliques allows to freely add one or two more rounds with the same time complexity. Moreover, when the key size of the cipher is larger than its block size, we show how to build the bicliques by an improved technique which does not require any additional data (on the contrary to previous biclique attacks). These techniques apply to PRESENT, DES, PRINCE and AES, improving the previously known results on these four ciphers. In particular, our attack on PRINCE applies to 8 rounds (out of 12), instead of 6 in the previous cryptanalyses. Some results are also given for theoretically estimating the sieving probability provided by some inputs and outputs of a given sbox.
\end{abstract}

Keywords: Meet-in-the-middle, bicliques, sbox, matching algorithms.

\section{Introduction}

Meet-in-the-middle (MITM) attacks are a widely used tool introduced by Diffie and Hellman in 1977. Through the years, they have been applied for analyzing the security of a substantial number of cryptographic primitives, including block ciphers, stream ciphers and hash functions, e.g. [20 5]12 15]14]. They exploit the fact that some internal state in the middle of the cipher can be computed both forwards from the plaintext and backwards from the ciphertext, and that none of these computations requires the knowledge of the whole master key. The attacker then only keeps the (partial) key candidates which lead to a collision in that internal state and discards all the other keys. This generic attack has drawn a lot of attention and raised many improvements, including the partial matching, where the computed internal states are not completely known, the technique of guessing some bits of the internal state [12, the all-subkeys approach [15], spliceand-cut 2313] and bicliques [18. The most popular application of bicliques is an

\footnotetext{
* Partially supported by the French Agence Nationale de la Recherche through the BLOC project under Contract ANR-11-INS-011.
}

R. Canetti and J.A. Garay (Eds.): CRYPTO 2013, Part I, LNCS 8042, pp. 222-240, 2013.

(C) International Association for Cryptologic Research 2013 
accelerated exhaustive search on the full AES [4. But, besides this degenerated application where the whole key needs to be guessed, short bicliques usually allow to increase the number of rounds attacked by MITM techniques without increasing the time complexity, but with a higher data complexity. Moreover, following [7, low-data attacks have attracted a lot of attention, motivated in part by the fact that, in many concrete protocols, only a few plaintext-ciphertext pairs can be eavesdropped. MITM attacks belong to this class of attacks in most cases (with a few exceptions like bicliques): usually, 1 or 2 known plaintext-ciphertext pairs are enough for recovering the key.

Our Contribution. This paper first provides a new generic improvement of MITM algorithms, named sieve-in-the-middle, which allows to attack a higher number of rounds. Instead of looking for collisions in the middle, we compute some input and output bits of a particular middle sbox $S$. The merging step of the algorithm then consists in efficiently discarding all key candidates which do not correspond to a valid transition through $S$. Intuitively, this technique allows to attack more rounds than classical MITM since it also covers the rounds corresponding to the middle sbox $S$ (e.g. two middle rounds if $S$ is a superbox). This new improvement is related to some previous results, including [2] where transitions through an ARX construction are considered; a similar idea was applied in [17] in a differential attack, and in [8] for side-channel attacks. This new generic improvement can be combined with bicliques, since short bicliques also allow to add a few rounds without increasing the time complexity. But, the price to pay is a higher data complexity. Here, we show that this increased data requirement can be avoided by constructing some improved bicliques, if the key size of the cipher is larger than its block size.

These new improvements and techniques are illustrated with four applications which improve previously known attacks. In Section 4, the sieve-in-the-middle algorithm combined with the improved biclique construction is applied to 8 rounds (out of 12) of PRINCE, with 2 known plaintext-ciphertext pairs, while the previous best known attack was on six rounds. Due to the page limitation, the other three applications are presented in the full version of this paper [9] only. In 9], we describe a sieve-in-the-middle attack on 8 rounds of PRESENT, which provides a very illustrative and representative example of our technique. This attack applies up to 8 rounds, while the highest number of rounds reached by classical MITM is only 6. A similar analysis on DES is presented in 9]; our attack achieves 8 rounds, while the best previous MITM attack (starting from the first one) was on 6 rounds. The cores of these two attacks have been implemented, confirming our theoretical analysis. In [9], we also show that we can slightly improve on some platforms the speed-up factor in the accelerated exhaustive search on the full AES performed by bicliques. The time complexity of the sieve-in-the-middle algorithm highly depends on the sieving probability of the middle sbox, i.e., on the proportion of pairs formed by a partial input and a partial output which correspond to a valid transition for $S$. We then give some results which allow to estimate the sieving probability of a given sbox. In particular, we show that the sieving probability is related to the branch number 
of the sbox, and we give a lower bound on the minimal number of known input and output bits which may provide a sieve.

\section{The Sieve-in-the-Middle Attack}

\subsection{Basic Idea}

The basic idea of the attack is as follows. The attacker knows one pair of plaintext and ciphertext $(P, C)$ (or several such pairs), and she is able to compute from the plaintext and from a part $K_{1}$ of the key candidate an $m$-bit vector $u$, which corresponds to a part of an intermediate state $x$. On the other hand, she is able to compute from the ciphertext and another part $K_{2}$ of the key candidate a $p$ bit vector $v$, which corresponds to a part of a second intermediate state $y$. Both intermediate states $x$ and $y$ are related by $y=S(x)$, where $S$ is a known function from $\mathbf{F}_{2}^{n}$ into $\mathbf{F}_{2}^{n^{\prime}}$, possibly parametrized by a part $K_{3}$ of the key. In practice, $S$ can be a classical sbox, a superbox or some more complex function, as long as the attacker is able to precompute and store all possible transitions between the input bits obtained by the forward computation and the output bits obtained backwards (or sometimes, these transitions can even be computed on the fly). In particular, the involved intermediate states $x$ and $y$ usually correspond to partial internal states of the cipher, implying that their sizes $n$ and $n^{\prime}$ are smaller than the blocksize.

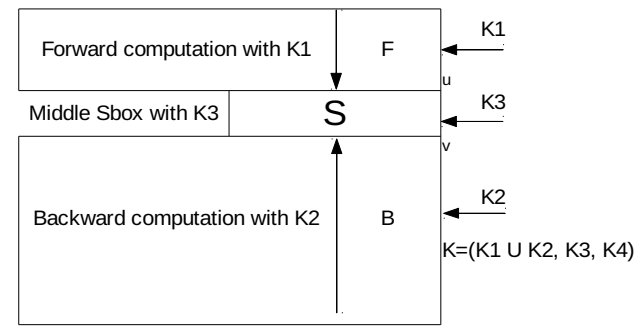

Fig. 1. Generic representation of Sieve-in-the-Middle

Then, the attacker is able to compute some pairs $(u, v)$ in $\mathbf{F}_{2}^{m} \times \mathbf{F}_{2}^{p}$ and she wants to determine whether those pairs can be some valid parts of a pair $(x, S(x))$ for some $x \in \mathbf{F}_{2}^{n}$ (and for some $K_{3}$ if $S$ depends on a part of the key). If it appears that no input $x \in \mathbf{F}_{2}^{n}$ can lead to a given $(u, v)$, then the keys $\left(K_{1}, K_{2}\right)$ from which $(u, v)$ has been obtained do not form a valid candidate for the key. In such a case, the $(m+p)$ positions corresponding to $(u, v)$ can be used as a sieve. The sieving probability is then the proportion of pairs $(u, v)$ corresponding to valid parts of $(x, S(x))$. Obviously, in classical MITM attacks, $u$ and $v$ correspond to the same $n$-bit part of an intermediate state and $S=\operatorname{Id}_{n}$; the sieving probability is then equal to $2^{-n}$. We now define precisely when a pair $(I, J)$ of input and output positions can be used as a sieve. 
Definition 1. Let $S$ be a function from $\mathbf{F}_{2}^{n}$ into $\mathbf{F}_{2}^{n^{\prime}}$. Let $I \subset\{1, \ldots, n\}$ and $J \subset$ $\left\{1, \ldots, n^{\prime}\right\}$ be two subsets with respective sizes $m$ and $p$. The sieving probability of $(I, J)$, denoted by $\pi_{I, J}$, is the proportion of all elements in $\mathbf{F}_{2}^{m+p}$ which can be written as $\left(x_{i}, i \in I ; S_{j}(x), j \in J\right)$ for some $x \in \mathbf{F}_{2}^{n}$. The pair $(I, J)$ is called an $(m, p)$-sieve for $S$ if $\pi_{I, J}<1$.

The smaller $\pi_{I, J}$, the better the sieving, because more candidates will be discarded. If $S$ depends on a $k_{3}$-bit value key $K_{3}$, the definition similarly applies but $S$ must be seen as a function with $\left(k_{3}+n\right)$ inputs.

When a large number of inputs and outputs of $S$ can be computed by the attacker, they can be used as a sieve, as shown in the following proposition.

Proposition 1. Any pair $(I, J)$ of sets of size $(m, p)$ with $m+p>n$ is a sieve for $S$ with sieving probability $\pi_{I, J} \leq 2^{n-(m+p)}$.

Proof. For any given $u$, there exists exactly $2^{n-m}$ values of $x$ such that $\left(x_{i}, i \in\right.$ $I)=u$. Thus, $\left(S_{j}(x), j \in J\right)$ can take at most $2^{n-m}$ different values, implying that $\pi_{I, J} \leq 2^{n-(m+p)}$.

However, smaller subsets $I$ and $J$ may provide a sieve even when $m+p \leq n$. This issue will be extensively discussed in Section 5 . More generally, $u$ and $v$ may consist of some information bits of $x$ and $y$, i.e., of some linear combinations of the bits of $x$ and $y$. We then define two linear functions $L: x \in \mathbf{F}_{2}^{n} \mapsto u \in \mathbf{F}_{2}^{m}$ and $L^{\prime}: y \in \mathbf{F}_{2}^{n} \mapsto v \in \mathbf{F}_{2}^{p}$. The corresponding sieving probability $\pi$ is now the proportion of $(u, v)$ such that there exists $x \in \mathbf{F}_{2}^{n}$ with $L(x)=u$ and $L^{\prime}(S(x))=v$. Then, $\pi$ can be seen as the sieving probability of $I=\{1, \ldots, m\}$ and $J=\{1, \ldots, p\}$ for the function $L^{\prime} \circ S \circ \widetilde{L}^{-1}$ where $\widetilde{L}$ is any linear permutation of $\mathbf{F}_{2}^{n}$ such that $\left(\widetilde{L}(x)_{i}, i \in I\right)=L(x)$.

\subsection{Description of the Attack}

We now precisely describe the improved MITM attack and provide its complexity. The secret key $K$ is divided into four (possibly non-disjoint) parts, $K_{1}$ to $K_{4} . K_{1}$ (resp. $K_{2}$ ) is the part of the key used in the forward (resp. backward) computation, while $K_{3}$ is involved in the middle $S$ function only (see Fig. 1). The key bits corresponding to $K_{4}$ are not involved in the MITM step. In the following, $k_{i}$ denotes the length of the key part $K_{i}$, while $k$ is the total key length. Moreover, $K_{1} \cap K_{2}$ denotes the bits shared by $K_{1}$ and $K_{2}$, and $\kappa$ corresponds to the size of this intersection.

We denote by $I$ (resp. $J$ ) the set of input positions of $S$ (resp. output positions) corresponding to $u$ (resp. $v$ ). The fact that a pair $(u, v)$ corresponds to a valid pair of inputs and outputs of $S$ is characterized by a Boolean relation $\mathcal{R}$ with $(m+p)$ inputs defined by

$$
\mathcal{R}(u, v)=1 \text { if and only if } \exists x \in \mathbf{F}_{2}^{n}:\left(x_{i}, i \in I\right)=u \text { and }\left(S(x)_{j}, j \in J\right)=v .
$$


The attack proceeds as follows.

for all $2^{\kappa}$ values of $K_{1} \cap K_{2}$ do

$\mathcal{L}_{f} \leftarrow \emptyset$ and $\mathcal{L}_{b} \leftarrow \emptyset$

// Forward computation

for all $2^{k_{1}-\kappa}$ values of the remaining bits of $K_{1}$ do compute $u=F_{K_{1}}(P)$ and add $u$ to $\mathcal{L}_{f}$

// Backward computation

for all $2^{k_{2}-\kappa}$ values of the remaining bits of $K_{2}$ do compute $v=B_{K_{2}}(C)$ and add $v$ to $\mathcal{L}_{b}$

// Merging step

Merge $\mathcal{L}_{f}$ and $\mathcal{L}_{b}$ w.r.t. Relation $\mathcal{R}$ and return the merged list $\mathcal{L}_{\text {sol }}$.

// Testing the remaining candidates

for all $K$ with $\left(K_{1}, K_{2}\right)$ in $\mathcal{L}_{\text {sol }}$ do

if $E_{K}(P)=C$ then

return $K$

Section 2.3 details some efficient algorithms for merging the two lists $\mathcal{L}_{f}$ and $\mathcal{L}_{b}$ (i.e. for recovering all the $(u, v)$ which satisfy $\mathcal{R}(u, v)=1$ ) with complexity lower than the product of their sizes.

With a Single Plaintext-Ciphertext Pair. Obviously, the whole secret key can be recovered only if the key length does not exceed the blocksize. Otherwise, $2^{k-b}$ possible keys will be returned in average where $b$ is the blocksize. The time complexity of the attack is given by:

$$
2^{\kappa}\left(2^{k_{1}-\kappa} c_{F}+2^{k_{2}-\kappa} c_{B}+C_{\text {merge }}\right)+\pi 2^{k} c_{E},
$$

where $\pi$ is the sieving probability of $(I, J)$ as defined in Definition 1, $c_{E}$ is the cost of one encryption, while $c_{F}$ and $c_{B}$ correspond to the costs of a partial encryption in the forward and backward directions. In most cases, $c_{F} \simeq c_{B} \simeq c_{E} / 2$. $C_{\text {merge }}$ is the time complexity of the merging step, and it depends on $k_{3}$. Its value is discussed in the following section. The average time complexity of the attack needs to be compared to $2^{k} c_{E}$ which is the cost of the exhaustive search. The memory complexity is mainly determined by the memory needed in the merging step. In some cases, it can be improved by storing only one among the two lists $\mathcal{L}_{f}$ and $\mathcal{L}_{b}$, when the auxiliary lists used in the merging step remain smaller.

With $N$ Plaintext-Ciphertext Pairs. If $N$ plaintext-ciphertext pairs are available to the attacker, then the average number of keys returned by the attack is $2^{k-N b}$, implying that the whole key will be recovered when $N \geq k / b$. The main modification in the attack concerns the last step where all key candidates in $\mathcal{L}_{\text {sol }}$ are tested: before performing an exhaustive search over $\left(K_{1} \cap K_{2}\right)$ and $K_{4}$ for testing all keys with $\left(K_{1}, K_{2}\right) \in \mathcal{L}_{\text {sol }}$, an additional sieving step is performed in order to reduce the size of $\mathcal{L}_{\text {sol }}$. Once a new solution $\left(K_{1}, K_{2}\right) \in \mathcal{L}_{\text {sol }}$ has been found, $(N-1)$ additional pairs $\left(u_{i}, v_{i}\right)$ generated from the other plaintextciphertext pairs are considered, and only the keys for which $\mathcal{R}\left(u_{i}, v_{i}\right)=1$ are 
kept in $\mathcal{L}_{\text {sol }}$ (note that, in some very particular situations, it might be more efficient to directly include in $\mathcal{L}_{f}$ and $\mathcal{L}_{b}$ the values $u$ and $v$ generated from several plaintext-ciphertext pairs, and then merge the lists). The average size of $\mathcal{L}_{\text {sol }}$ after this additional sieving step is then $\pi^{N} 2^{k_{1}+k_{2}-2 \kappa}$. But this formula should be adapted to the case where $S$ depends on a part of the secret key $K_{3}$ : indeed the merging step determines a candidate for $\left(K_{1}, K_{2}, K_{3}\right)$. Then, the sieving probability of the additional sieving step $\pi^{\prime}$ differs from $\pi$ since the value of $K_{3}$ is now fixed. $\pi^{\prime}$ is then the sieving probability of $(I, J)$ for $S_{K_{3}}$ averaged over all $K_{3}$. Then, in the case of $N$ plaintext-ciphertext pairs, the cost of the forward and backward computations are multiplied by $N$, while the cost of the testing part decreases:

$$
2^{\kappa}\left(N 2^{k_{1}-\kappa} c_{F}+N 2^{k_{2}-\kappa} c_{B}+C_{\text {merge }}\right)+\pi\left(\pi^{\prime}\right)^{N-1} 2^{k} c_{E} .
$$

\subsection{Merging the Two Lists Efficiently}

Very often, the middle function $S$ can be decomposed into several smaller sboxes, and the merging step can be performed group-wise. The problem of merging two large lists with respect to a group-wise Boolean relation has been defined and addressed by Naya-Plasencia in [19, Section 2]. Here, we focus on three algorithms proposed in [19], namely instant matching, gradual matching and an improvement of the parallel matching due to 10 . We provide general and precise formulas for the average time and memory complexities of these three algorithms. Actually, in our case, the lists to be merged may be small. Then, the construction of some auxiliary tables, which had a negligible cost in [19] for large lists, must now be taken into account. It might even become the bottleneck of the algorithm. Thus, when the involved lists are small, it is harder to determine a priori which algorithm is the most efficient in a given case. Then, in each application, we need to check thoroughly which algorithm provides the best complexity. The optimal case may even sometimes correspond to the combination of two algorithms.

In the following, we consider two lists, $\mathcal{L}_{A}$ of size $2^{\ell_{A}}$ and $\mathcal{L}_{B}$ of size $2^{\ell_{B}}$, whose roles are interchangeable. The elements of both lists can be decomposed into $t$ groups: the $i$-th group of $a \in \mathcal{L}_{A}$ has size $m_{i}$, while the $i$-th group of $b \in \mathcal{L}_{B}$ has size $p_{i}$. The Boolean relation $\mathcal{R}$ can similarly be considered group-wise: $\mathcal{R}(a, b)=1$ if and only $\mathcal{R}_{i}\left(a_{i}, b_{i}\right)=1$ for all $1 \leq i \leq t$. The sieving probability $\pi$ associated to $\mathcal{R}$ then corresponds to the product of the sieving probabilities $\pi_{i}$ associated to each $\mathcal{R}_{i}$. Since each $\mathcal{R}_{i}$ corresponds to an sbox $S_{i}$ with $n_{i}$-bit inputs, a table storing all $\left(a_{i}, b_{i}\right)$ such that $\mathcal{R}_{i}\left(a_{i}, b_{i}\right)=1$ can be built with time complexity $2^{n_{i}}$, by computing all $\left(x_{i}, S_{i}\left(x_{i}\right)\right), x_{i} \in \mathbf{F}_{2}^{n_{i}}$. The corresponding memory complexity is proportional to $\pi_{i} 2^{m_{i}+p_{i}}$. This cost won't be included in the cost of the merging algorithm since, in the sieve-in-the-middle process, the tables will be built once for all and not $2^{\kappa}$ times. As we will see, in some situations, these tables can be built "on-the-fly" with much fewer operations.

A complete description of the three matching algorithms is provided in the full version [9]. It is worth noticing that the size of the list $\mathcal{L}_{\text {sol }}$ returned by the matching algorithm is not included in the memory complexity since each of its elements can be tested in the attack as soon as it has been found. 
Instant Matching. Instant matching successively considers all elements $\mathcal{L}_{B}$ : for each $b \in \mathcal{L}_{B}$, a list $\mathcal{L}_{a u x}$ of all $a$ such that $\mathcal{R}(a, b)=1$ is built, and each element of $\mathcal{L}_{\text {aux }}$ is searched within $\mathcal{L}_{A}$. Its complexity is

$$
\text { Time }=\pi 2^{\ell_{B}+m}+\pi 2^{\ell_{A}+\ell_{B}} \text { and Memory }=2^{\ell_{A}}+2^{\ell_{B}} .
$$

Gradual Matching. Gradual matching is a recursive procedure: all elements are decomposed into two parts, the first $t^{\prime}$ groups and the last $\left(t-t^{\prime}\right)$, with $t^{\prime}<t$. For each possible value $\beta$ of the first $t^{\prime}$ groups, the sublist $L_{B}(\beta)$ is built. It consists of all elements in $\mathcal{L}_{B}$ whose first $t^{\prime}$ groups take the value $\beta$. Now, for each $\alpha$ such that $\mathcal{R}_{i}\left(\alpha_{i}, \beta_{i}\right)=1,1 \leq i \leq t^{\prime}, L_{B}(\beta)$ is merged with the sublist $L_{A}(\alpha)$ which consists of all elements in $\mathcal{L}_{A}$ whose first $t^{\prime}$ groups take the value $\alpha$. Then, we need to merge two smaller lists, of respective sizes $2^{\ell_{A}-\sum_{i=1}^{t^{\prime}} m_{i}}$ and $2^{\ell_{B}-\sum_{i=1}^{t^{\prime}} p_{i}}$.

$$
\text { Time }=\left(\prod_{i=1}^{t^{\prime}} \pi_{i}\right) 2^{\sum_{i=1}^{t^{\prime}} m_{i}+p_{i}} C_{\text {merge }} \text { and Memory }=2^{\ell_{A}}+2^{\ell_{B}} .
$$

where $C_{\text {merge }}$ is the cost of merging the two remaining sublists.

Parallel Matching without Memory. We give here the first general description of the memoryless version of parallel matching. This algorithm applies an idea from [10] to the parallel matching algorithm from [19]: instead of building a big auxiliary list as in the original parallel matching, we here build small ones which do not need any additional memory. In parallel matching, the elements in both lists are decomposed into three parts: the first $t_{1}$ groups, the next $t_{2}$ groups, and the remaining $\left(t-t_{1}-t_{2}\right)$ groups. Both lists $\mathcal{L}_{A}$ and $\mathcal{L}_{B}$ are sorted in lexicographic order. Then, $\mathcal{L}_{A}$ can be seen as a collection of sublists $\mathcal{L}_{A}(\alpha)$, where $\mathcal{L}_{A}(\alpha)$ is composed of all elements in $\mathcal{L}_{A}$ whose first $t$ groups equal $\alpha$. Similarly, $\mathcal{L}_{B}$ is seen as a collection of $\mathcal{L}_{B}(\beta)$. The matching algorithm then proceeds as follows. For each possible value $\alpha$ for the first $t$ groups, an auxiliary list $\mathcal{L}_{\text {aux }}$ is built, corresponding to the union of all $\mathcal{L}_{B}(\beta)$ where $(\alpha, \beta)$ satisfies the first $t$ relations $\mathcal{R}_{j}$. The list $\mathcal{L}_{\text {aux }}$ is sorted by its next $t_{2}$ groups. Then, for each element in $\mathcal{L}_{A}(\alpha)$, we check if a match for its next $t_{2}$ groups exists in $\mathcal{L}_{\text {aux }}$. For each finding, the remaining $\left(t-t_{1}-t_{2}\right)$ groups are tested and only the elements which satisfy the remaining $\left(t-t_{1}-t_{2}\right)$ relations are returned. Details on the evaluation of the time and memory complexities are given in 9 .

$$
\begin{gathered}
\text { Time }=\left(\prod_{i=1}^{t_{1}} \pi_{i}\right) 2^{\ell_{B}+\sum_{i=1}^{t_{1}} m_{i}}+\left(\prod_{i=t_{1}+1}^{t_{1}+t_{2}} \pi_{i}\right) 2^{\ell_{A}+\sum_{i=t_{1}+1}^{t_{1}+t_{2}} p_{i}}+\left(\prod_{i=1}^{t_{1}+t_{2}} \pi_{i}\right) 2^{\ell_{A}+\ell_{B}} \\
\text { Memory }=2^{\ell_{A}}+2^{\ell_{B}}+\left(\prod_{i=1}^{t_{1}} \pi_{i}\right) 2^{\ell_{B}} .
\end{gathered}
$$




\section{Combining Sieve-in-the-Middle and Bicliques}

Sieve-in-the-middle, as a generic technique, can be combined with other improvements of MITM attacks, in particular with bicliques [418. The general purpose of bicliques is to increase the number of rounds attacked by MITM techniques. Here, we briefly describe how bicliques can increase the number of rounds attacked by the previously described sieve-in-the-middle algorithm. This can be done at no computational cost, but requires a higher data complexity. In order to avoid this drawback, we then present an improvement of bicliques which applies when the key length exceeds the block size of the cipher.

\subsection{Sieve-in-the-Middle and Classical Bicliques}

The combination of both techniques is depicted on Figure 2 the bottom part is covered by bicliques, while the remaining part is covered by a sieve-in-the-middle algorithm. In the following, $H_{K_{8}}: X \mapsto C$ denotes the function corresponding to the bottom part of the cipher, and $K_{8}$ represents the key bits involved in this part. Then, $K_{8}$ is partitioned into three disjoint subsets, $K_{5}, K_{6}$ and $K_{7}$. The value taken by $K_{i}$ with $5 \leq i \leq 7$ will be represented by an integer in $\left\{0, \ldots, 2^{k_{i}}-1\right\}$. A biclique can be built if the active bits in the computation of $H_{K_{8}}(X)$ when $K_{6}$ varies and the active bits in the computation of $H_{K_{8}}^{-1}(C)$ when $K_{5}$ varies are two disjoint sets. In this case, an exhaustive search over $K_{7}$ is performed and a biclique is built for each value $h$ of $K_{7}$ as follows. We start from a given ciphertext $C^{0}$ and a chosen key $K_{8}^{0}=(0,0, h)$ formed by the candidate for $K_{7}$ and the zero value for $K_{5}$ and $K_{6}$. We compute $X_{h}^{0}=H_{0,0, h}^{-1}\left(C^{0}\right)$. Next, we compute backwards from $C^{0}$ the intermediate state $X_{h}^{i}=H_{i, 0, h}^{-1}\left(C^{0}\right)$ for each possible value $i$ for $K_{5}$. Similarly, we compute forwards from $X_{h}^{0}$ the ciphertext $C_{h}^{j}=H_{0, j, h}\left(X_{h}^{0}\right)$ for each possible value $j$ of $K_{6}$. Since the two differential paths are independent, we deduce that $H_{i, j, h}\left(X_{h}^{i}\right)=C_{h}^{j}$ for all values $(i, j)$ of $\left(K_{5}, K_{6}\right)$.

Then, the sieve-in-the-middle algorithm can be applied for each $K_{7}$ and each value for $\left(K_{1} \cap K_{2}\right)$. The list $\mathcal{L}_{b}$ of all output vectors $v$ is computed backwards

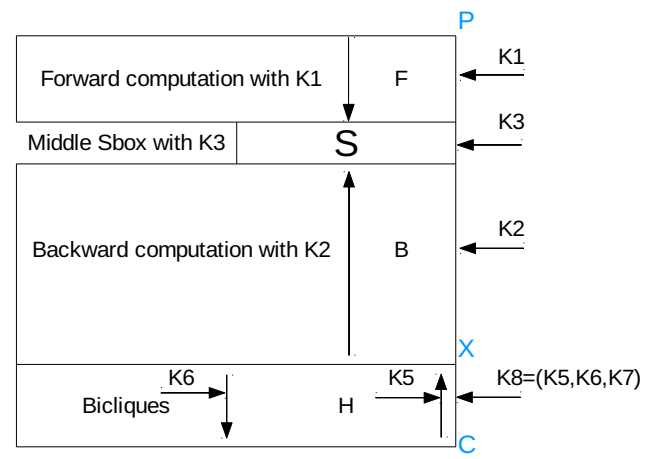

Fig. 2. Generic representation of Sieve-in-the-Middle and bicliques 
from $X_{h}^{i}$ for each value $i$ of $K_{5}$ and each value of $K_{2} \backslash\left(K_{1} \cap K_{2}\right)$. The list $\mathcal{L}_{f}$ of all input vectors $u$ is computed forwards from all plaintexts $P_{h}^{j}$ corresponding to $C_{h}^{j}$ for each value $j$ of $K_{6}$ and each value of $K_{1} \backslash\left(K_{1} \cap K_{2}\right)$. We then merge those two lists of respective sizes $2^{\left|K_{2} \cup K_{5}\right|}$ and $2^{\left|K_{1} \cup K_{6}\right|}$.

As in classical MITM with bicliques, the decomposition of $K_{8}$ should be such that the bits of $K_{5}$ do not belong to $K_{1}$, the bits of $K_{6}$ do not belong to $K_{2}$ and the bits of $K_{7}$ should lie in $\left(K_{1} \cap K_{2}\right)$. The best strategy here seems to choose $\left(K_{5}, K_{6}\right)$ such that the bits of $K_{5}$ belong to $K_{2} \backslash\left(K_{1} \cap K_{2}\right)$, and the bits of $K_{6}$ belong to $K_{1} \backslash\left(K_{1} \cap K_{2}\right)$. In this case, we have to add to the time complexity of the attack the cost of the construction of the bicliques, i.e., $2^{k_{7}}\left(2^{k_{5}}+2^{k_{6}}\right) c_{H}$ (very rarely the bottleneck), where $c_{H}$ is the cost of the partial encryption or decryption corresponding to the rounds covered by the bicliques. The main change is that the data complexity has increased since the attack now requires the knowledge of all plaintext-ciphertext pairs $\left(P_{h}^{j}, C_{h}^{j}\right)$ corresponding to all possible values $(j, h)$ for $\left(K_{6}, K_{7}\right)$. The data complexity then would correspond to $2^{k_{6}+k_{7}}$ pairs of plaintext-chosen ciphertexts, but it is usually smaller since the ciphertexts $C_{h}^{j}$ only differ on a few positions.

\subsection{Improved Bicliques for Some Scenarios}

Now, we describe a generic idea for improving bicliques in certain scenarios and reducing the data complexity to a single plaintext-ciphertext pair. Our improvement usually applies when the total key size of the cipher is larger than the block size. This occurs for instance when whitening keys are used. A detailed and successful application is demonstrated on PRINCE in Section 4. The main idea of our improvement is to gather some parts of the partial exhaustive search over $K_{7}$ into different groups such that, within a group, all obtained ciphertexts $C^{j}$ are equal to $C^{0}$.

We consider a biclique repartition of keys consistent with the sieve-in-themiddle part: we choose $K_{5} \subset K_{2} \backslash\left(K_{1} \cap K_{2}\right)$ as previously, and some set $K_{6}^{\prime} \subset K_{1}$ (this differs from the classical biclique construction where we had $K_{6} \subset K_{1} \backslash\left(K_{1} \cap\right.$ $\left.K_{2}\right)$ ). Let $\Delta_{6}^{C}$ be the positions of the bits of $C$ which may be affected by $K_{6}^{\prime}$ when computing forward from $X$, and let $\Delta_{6}^{X}$ be the positions of the bits of $X$ which may be affected by $\Delta_{6}^{C}$ and $K_{6}^{\prime}$ during the backward computation. In classical bicliques, the path generated in the backward direction by the different $K_{5}$ must be independent from the path generated in the forward direction by the different $K_{6}^{\prime}$. Here, we also require this first path generated by $K_{5}$ to be independent from the backward path generated when the ciphertext bits in positions $\Delta_{6}^{C}$ vary. For instance, in the example depicted on Figure 3, $H$ follows the Even-Mansour construction, i.e., it is composed of an unkeyed permutation $H^{\prime}$ and the addition of two whitening keys $K_{a}$ and $K_{b}$. The positions of $K_{5}$ and $K_{6}^{\prime}$ are represented in red and blue respectively, and it can be checked that the corresponding paths are independent.

In this situation, an improved biclique without any additional data can be built if the size of $\Delta_{6}^{X}$ is smaller than $k_{6}^{\prime}$. In our context, the algorithm has to be repeated for each value $h$ for $K_{7}^{\prime}=K_{8} \backslash\left(K_{5} \cup K_{6}^{\prime}\right)$, but the index $h$ will 


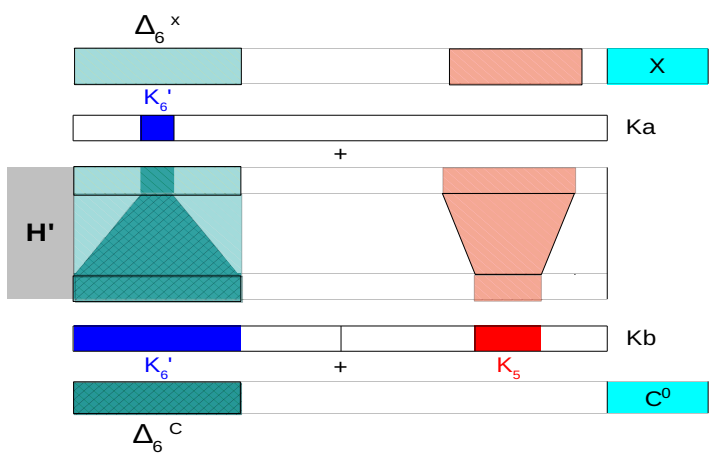

Fig. 3. Example of the improved biclique construction

be omitted in the description. First, we precompute the values obtained from a chosen $C^{0}$ when $K_{6}^{\prime}$ takes all possible values. If the number of information bits in $\Delta_{6}^{X}$ is less than $k_{6}^{\prime}$, all $2^{k_{6}^{\prime}}$ transitions can be represented by several lists $\mathcal{L}_{j}$, each containing the different values of $K_{6}^{\prime}$ which all map $C^{0}$ to the same value of the state $X, X_{j}$ (see Figure 4(a). For the sake of simplicity, we assume that all these lists have the same size $2^{\ell}$. In most cases, we have $\ell=k_{6}^{\prime}-\left|\Delta_{6}^{X}\right|$. For the example depicted on Figure 3, we assume that $H^{\prime}$ is such that the function obtained by restricting its inputs to the positions in $\Delta_{6}^{X}$ and its outputs to the positions in $\Delta_{6}^{C}$ is a permutation. Then, it clearly appears that the number of bits in $\Delta_{6}^{X}$ is equal to the number of bits of $K_{6}^{\prime} \cap K_{b}$, and thus strictly smaller than the number of bits of $K_{6}^{\prime}$. More precisely, there are exactly $2^{\ell}$ values of $K_{6}^{\prime}$, with $\ell=\left|K_{6}^{\prime} \cap K_{a}\right|$, which provide the same value of $X=H^{\prime-1}\left(C^{0}+K_{b}\right)+K_{a}$ when $K_{6}^{\prime}$ varies and all other bits are fixed.

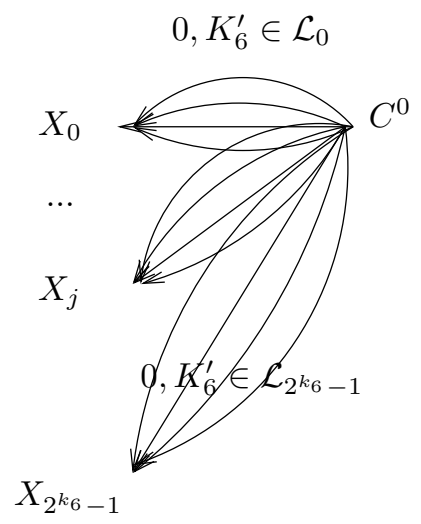

(a) Step 1

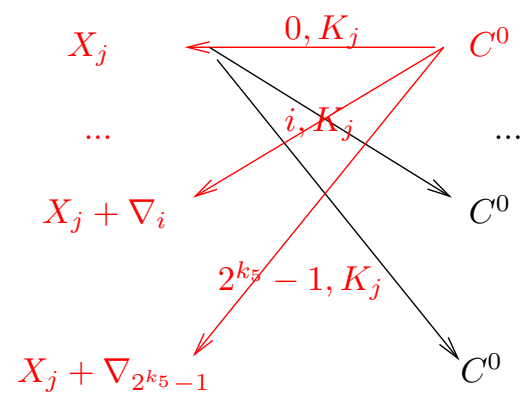

(b) Step 2: to be repeated for the $2^{k_{6}^{\prime}-\ell}$ values of $j$

Fig. 4. Improved biclique construction 
Now, for each of the $2^{k_{6}^{\prime}-\ell}$ values of $X_{j}$, all transitions from $C^{0}$ to $X_{j}$ through different values of $K_{6}^{\prime} \in \mathcal{L}_{j}$ can also be seen as the $2^{\ell}$ biclique transitions from $X_{j}$ to $C^{0}$ through some particular values of the key $K_{6}^{\prime}$ (these transitions are represented in black on Figure 4(b)).

Now, the second step consists in building the bicliques in the other direction: from $C^{0}$ for each value of $X_{j}$. For each of the $2^{k_{6}^{\prime}-\ell}$ values of $j$, we fix the value of $K_{6}^{\prime}$ to a constant value $K_{j}$ appearing in $\mathcal{L}_{j}$. This way, the part of $X$ corresponding to $\Delta_{6}^{X}$ is the same for all the transitions of the bicliques, and this property holds even when $K_{5}$ is modified since both corresponding paths are independent. We then consider the $2^{k_{5}}$ possible values $i$ for $K_{5}$ and compute the corresponding $X=X_{j}+\nabla_{i}$ (see Figure 4(b)). We then deduce the $2^{k_{5}+k_{6}^{\prime}}$ transitions $H\left(X_{j}+\nabla_{i}\right)_{\left(i, K_{6}^{\prime}\right)}=C^{0}$ for all $K_{6}^{\prime} \in \mathcal{L}_{j}$, from $\left(2^{k_{6}^{\prime}}+\right.$ $\left.2^{k_{6}^{\prime}-\ell+k_{5}}\right)$ computations of the function. Indeed, the first term in the complexity corresponds to the precomputation phase (Step 1), and the second one to the number of lists $\mathcal{L}_{j}, 2^{k_{6}^{\prime}-\ell}$, multiplied by the cost for building the bicliques in the other direction. The main advantage of this construction is that it can be combined with the sieve-in-the-middle part as previously described, but it now requires a single plaintext-ciphertext pair, the one formed by $\left(P^{0}, C^{0}\right)$.

Finally, we assume that the bits of $K_{5}$ belong to $K_{2} \backslash\left(K_{1} \cap K_{2}\right)$, the bits of $K_{6}^{\prime}$ belong to $K_{1}$ and the bits of $K_{7}^{\prime}$ are the bits from $\left(K_{1} \cup K_{2}\right) \backslash\left(K_{5} \cup K_{6}^{\prime}\right)$, the time complexity of the attack is:

$$
2^{k_{7}^{\prime}}\left(2^{k_{6}^{\prime}}+2^{k_{6}^{\prime}-\ell+k_{5}}\right) c_{H}+2^{k_{1}} c_{F}+2^{k_{2}} c_{B}+2^{\kappa} C_{\text {merge }}+\pi 2^{k} c_{E}
$$

where $C_{\text {merge }}$ is the cost of merging the lists of size $2^{k_{1}-\kappa}$ and $2^{k_{2}-\kappa}$ with respect to the sieving conditions.

A similar idea can also be used for choosing an appropriate $K_{5}$ which delays the propagation of the unknown bits during the forward computation. This will be shown in the case of Prince.

\section{Application to PRINCE}

PRINCE is a lightweight block cipher designed by Borghoff et al. [6]. Though being very recent, it has already waked the interest of many cryptanalysts [21|16|1]. The best known attacks so far on the proposed cipher, including the security analysis performed by the authors, reach 6 rounds. In particular, MITM with bicliques (without guessing the whole key) is said to reach at most 6 rounds (out of 12). In 16, a reduction of the security by one bit is presented, and in 1] an accelerated exhaustive search using bicliques is presented. Here, we describe how to build sieve-in-the-middle attacks on 8 rounds with data complexity 1 (or 2 if we want to the whole key instead of a set of candidates). In addition to the new sieve-in-the-middle technique, we use the improved method for constructing bicliques presented in Section 3.2 . 


\subsection{Brief Description of PRINCE}

PRINCE operates on 64-bit blocks and uses a 128-bit key composed of two 64-bit elements, $K_{a}$ and $K_{b}$. Its structure is depicted on Figure 5. PRINCE is based on the so-called FX-construction: two whitening keys $W_{i n}=\left(K_{a}+K_{b}\right)$ and $W_{\text {out }}=\left(K_{a}^{\prime}+K_{b}\right)$ are xored respectively to the input and to the output of a 12-round core cipher parametrized by $K_{b}$ only. The value of $K_{a}^{\prime}$ involved in the post-whitening key is derived from $K_{a}$ by $K_{a}^{\prime}=\left(K_{a} \ggg 1\right) \oplus\left(K_{a} \gg 63\right)$.

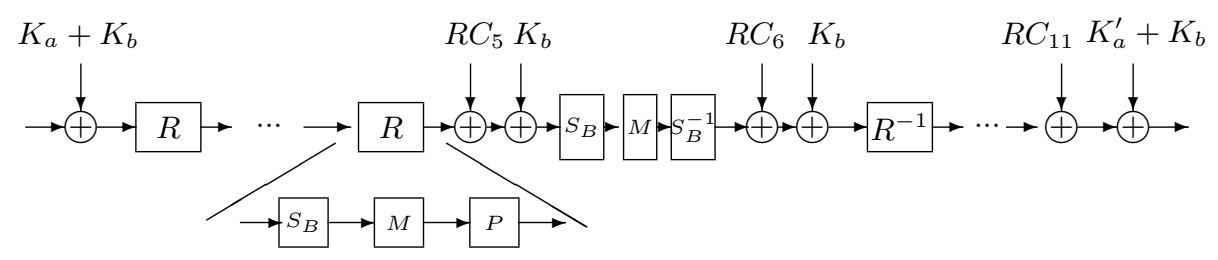

Fig. 5. Structure of PRINCE

The round function is composed of:

- a non-linear layer $S_{B}$ corresponding to 16 parallel applications of a $4 \times 4$ $\operatorname{sbox} \sigma$.

- a linear layer $P \circ M$, where $M$ is the parallel application of 4 involutive mixcolumns operations on 16 bits each (defined either by $\hat{M}^{(0)}$ or by $\hat{M}^{(1)}$ ). This transformation is then followed by a permutation $P$ of the 16 nibbles which is the same as the ShiftRows transformation used in the AES.

- the addition of a round constant $R C_{i}$ and of the subkey $K_{b}$.

The first 5 rounds in PRINCE correspond to the previous round permutation $R$, while the last 5 rounds are defined by the inverse permutation $R^{-1}$. The two middle rounds correspond to the successive applications of $S_{B}, M$ and $S_{B}^{-1}$.

\subsection{Sieve-in-the-Middle and Improved Bicliques on 8 Rounds}

Sieve-in-the-Middle on Six Rounds. We first describe the sieve-in-themiddle part of the attack, which covers Rounds 1 to 6 (see Figure 6). The internal state $X$ after Round 6 is supposed to be known, as well as the plaintext. The sieving step is done with respect to a function $S$ which covers Round 3 and the $S_{B}$ level of Round 4. This middle function $S$ can then be decomposed as four $16 \times 16$ superboxes: the colored nibbles in the middle of Figure 6 represent the nibbles belonging to the same superbox.

The 128 keybits in PRINCE are then decomposed as depicted on Figure 7

- $K_{1}$, i.e. the keybits known in the forward direction, are represented in white and in blue in $K_{b}$ and the first whitening key $W_{i n}$. They correspond to all bits $K_{b}$ and $W_{i n}$ except the 11 leftmost bits of the third 16-bit group in $K_{b}$. 

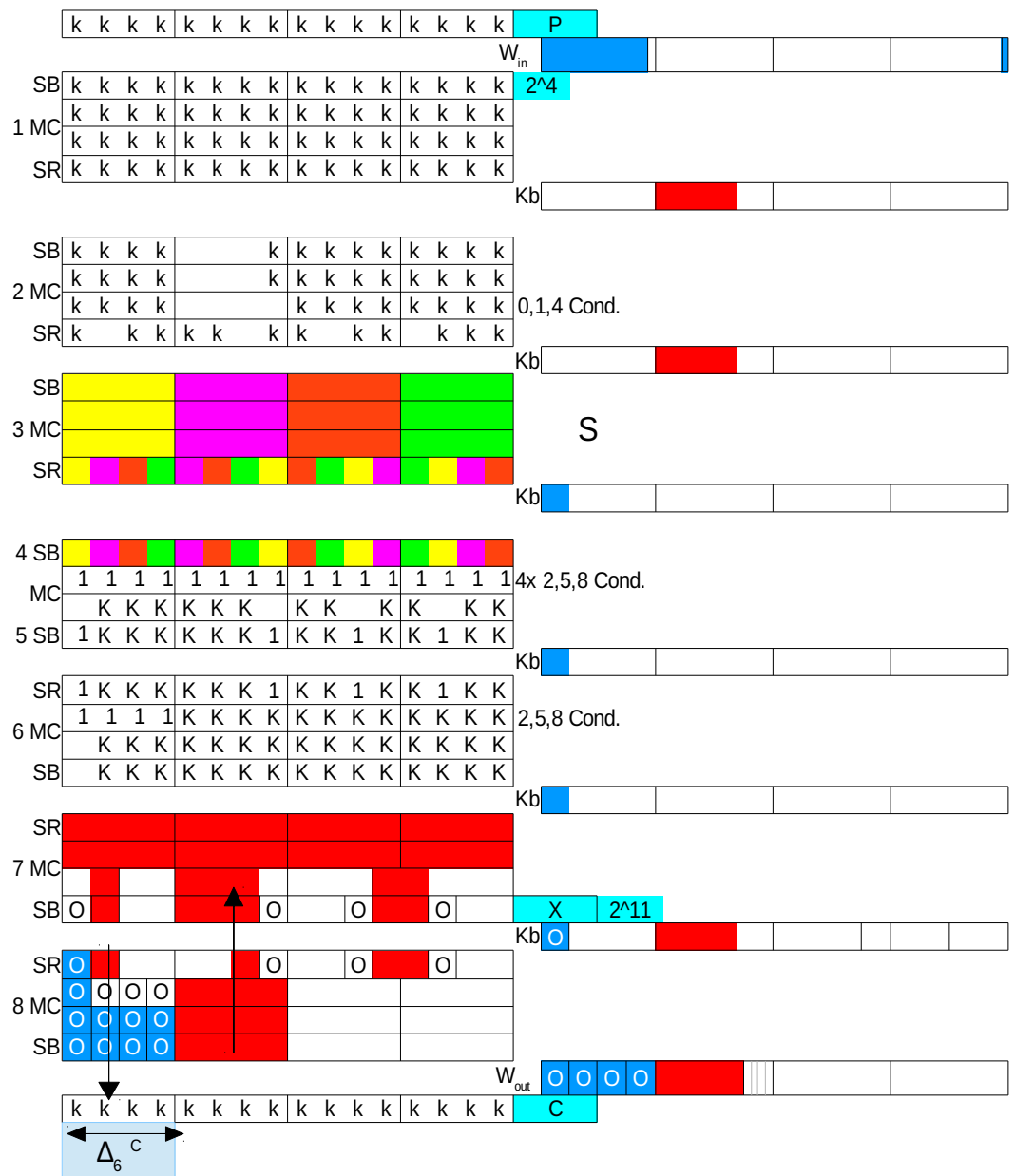

Fig. 6. Sieve-in-the-middle attack on 8 rounds of PRINCE with data complexity of 1

- $K_{2}$, i.e. the keybits known in the backward direction, are represented in white and in red in $K_{b}$ and $W_{i n}$. They correspond to all bits of $K_{b}$ and $W_{i n}$ except the leftmost nibble of $K_{b}$ and the 16 bits at positions 0 and 49 to 63 in $W_{i n}$.

It follows that the intersection $\left(K_{1} \cap K_{2}\right)$ consists of $\kappa=97$ information bits of $\left(K_{a}, K_{b}\right)$ : the 49 white bits in $K_{b}$ and the 48 white bits in $W_{i n}$.

The algorithm is described on Figure 6, where each nibble which contains ' $\mathrm{K}$ ' is known in the backward computation, each nibble which contains ' $\mathrm{k}$ ' is known in the forward computation and ' 1 ' means that there is a known bit in the nibble. The right part of the figure represents the key. We will exploit the fact that, for each $16 \times 16$ mixcolumns operation, there exist 4 output bits (one 


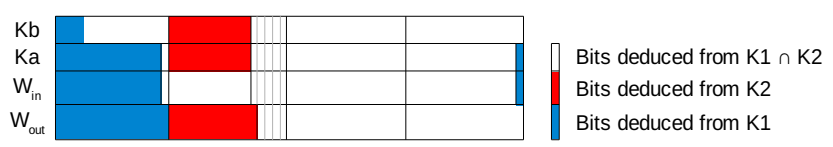

Fig. 7. Decomposition of the key in the attack on 8 rounds of PRINCE. $W_{i n}=K_{a} \oplus K_{b}$ and $W_{\text {out }}=\left(K_{a} \ggg 1\right) \oplus\left(K_{a} \gg 63\right) \oplus K_{b}$.

per nibble), as well as 8 information bits of the output, which do not depend on a given input nibble. Each of these 8 information bits corresponds to the sum of two output bits (see [9, Sect. 6.2] for details). In the backward computation, from State $X$ and $K_{2}$, we can compute 3 nibbles of each input of the mixcolumns operations at Round 5 . Then, we deduce one bit in each nibble of the output of the middle function $S$, as well as 32 information bits which involve the outputs of two different superboxes. When considering $s<4$ superboxes together, the number of information bits known is reduced to 8 if $s=2$, and to 20 if $s=3$.

In the forward computation, from the plaintext $P$ and $K_{1}$, we compute three input nibbles of each superbox. From the mixcolumns operation in Round 2 whose input is partially known, we can also have 4 additional information bits on the input of the middle function $S$. When considering $s<4$ superboxes together, the number of information bits known is reduced to 0 if $s=2$ and to 1 if $s=3$.

Then, we need to merge the two lists $\mathcal{L}_{f}$ and $\mathcal{L}_{b}$ of respective sizes $2^{4}$ and $2^{11}$. Since $m=4 \times 12+4=52$ input bits and $p=4 \times 4+32=48$ output bits are known, the total sieving probability $\pi$ is at most $2^{64-(52+48)}=2^{-36}$. In the following, the tables $T_{i}$ providing all transitions for the four superboxes $S_{j}$ are supposed to be known 1 .

We are going to first apply the instant matching on the first two blocks (orange and green), i.e., instant matching as described in [9, Algo. 1] with parameters $n_{1}=n_{2}=16$ and $m_{1}=m_{2}=12$ and $p_{1}+p_{2}=8+8=16$. The sieving probability of these two superboxes together is then $\pi_{1,2}=2^{32-(24+16)}=2^{-8}$. We consider $\mathcal{L}_{A}=\mathcal{L}_{b}$ and $\mathcal{L}_{B}=\mathcal{L}_{f}$. From the corresponding formula in Section 2.3. we get that the time complexity of this step is $2^{-8} 2^{4+16}+2^{-8} 2^{15} \approx 2^{12}$. With this complexity we have found $2^{15} \pi_{1,2}=2^{7}$ input-output pairs of $S$ which are valid for the first two superboxes. We can now check whether each of these pairs is also valid for the two remaining superboxes. Now, the sieving probability for the remaining part is at most $2^{-36} \times 2^{+8}=2^{-28}$ as the total sieving probability is at most $2^{-36}$.

Therefore, at the end of the merging step, for each guess of the $\kappa=113$ bits of $\left(K_{1} \cap K_{2}\right)$, we have a probability of $2^{7-28}=2^{-21}$ of finding a correct configuration for the 15 remaining bits of $\left(K_{1}, K_{2}\right)$. This means that the testing step will consider $2^{113-21}=2^{92}$ keys, and it will recover $2^{64}$ possible candidates

\footnotetext{
${ }^{1}$ The orange and green superboxes that involve common key bits only can be computed on the fly and will be used first for the instant matching. For each pair we obtain, the whole key is already known, so we can repeat the on-the-fly procedure.
} 
for the whole key. If two plaintext-ciphertext pairs are available, the testing step will consider $2^{92-36}=2^{56}$ keys instead of $2^{92}$, leading to performing a test over $2^{56}$ candidates for recovering the correct key.

Improved Bicliques Part. Our attack combines the previous sieve-in-themiddle algorithm with bicliques built as described in Section 3.2, without increasing the data complexity. We define $K_{6}^{\prime}$ as the five nibbles corresponding to the union of the leftmost nibble of $K_{b}$ and the four leftmost nibbles of the whitening key $W_{\text {out }}=\left(K_{a}^{\prime}+K_{b}\right)$. Then, $\Delta_{6}^{C}$ is represented on Figure 6 by the four 'O' symbols in the line before $C$. Also, $\Delta_{6}^{X}$ then corresponds to the 'O' symbols in $X$. Then, $\left|\Delta_{6}^{C}\right|=16$ and $\left|\Delta_{6}^{X}\right|=16$. The remaining 'O' show the path from $\Delta_{6}^{C}$ to $\Delta_{6}^{X}$. All $2^{20}$ transitions obtained when $K_{6}^{\prime}$ varies correspond, for each one of the $2^{16}$ possible values of $j$, to $2^{4}$ biclique transitions from $X_{j}$ to $C$. Then, $K_{5}$ is defined as the 11 leftmost bits of the third 16-bit group of $K_{b}$, implying that $K_{5}$ is equal to $K_{2} \backslash\left(K_{1} \cap K_{2}\right)$. The path generated in the backward direction, represented in red, is then independent from the blue path generated by $K_{6}^{\prime}$, and also from the path with 'O' symbols from $\Delta_{6}^{C}$ to $\Delta_{6}^{X}$.

The complete algorithm then consists in performing an exhaustive search over the $\kappa=97$ common bits corresponding to the white bits of $K_{b}$ and $W_{i n}$ in Figure 7. The previously described bicliques determine $2^{16}$ states $X_{j}$, and $2^{4}$ transitions from each $X_{j}$ to $C$. Then, for each $X_{j}$, we examine the corresponding $2^{4}$ values of $K_{6}^{\prime}$. For those $K_{6}^{\prime}$, we compute forwards from the plaintext $P$ the list of all $2^{4}$ vectors $u$. It is worth noticing that even if the red bits of $K_{a}$ and $K_{b}$ are unknown in the forward direction, their sum is known (see Fig. (7). Similarly, the list $\mathcal{L}_{b}$ of all vectors $v$ is computed backwards from the $2^{11} X^{i}$ and their associated value $i$ for $K_{5}$. From the formula given in Section 3.2 , we deduce that, for one plaintext-ciphertext pair, the time complexity is

Time $=2^{97}\left(2^{20}+2^{16+11}\right) c_{H}+2^{117} c_{F}+2^{113} c_{B}+2^{97} \times 2^{12}+2^{-36} \times 2^{128} c_{E} \simeq 2^{124} c_{H}$.

We have then gained more than four bits over the exhaustive search $\left(2^{128} c_{E}\right)$. The memory complexity is of $2^{20}$, corresponding to the precomputed table in the construction of the improved bicliques, since the transition tables for the superboxes can be computed on the fly.

\section{Sieving Probability and Related Properties of the Sbox}

\subsection{General Properties}

In this section, we focus on the general problem of theoretically estimating the sieving property provided by two subsets $I \subset\{1, \ldots, n\}$ and $J \subset\left\{1, \ldots, n^{\prime}\right\}$, with respective sizes $m$ and $p$, for a given function $S$ from $\mathbf{F}_{2}^{n}$ into $\mathbf{F}_{2}^{n^{\prime}}$. In particular, we provide some results on the minimal value of $(m+p)$ for which a sieve exists. In the following, $S_{J}$ denotes the function from $\mathbf{F}_{2}^{n}$ into $\mathbf{F}_{2}^{p}$ corresponding to the $p$ coordinates of $S$ defined by $J$. Also, for any affine subspace $W, S_{\mid W}$ denotes the restriction of $S$ to $W$, i.e., the function defined on $W$ by $S_{\mid W}(x)=S(x)$. Obviously, $S_{\mid W}$ can be identified with a function of $\operatorname{dim} W$ input variables. 
For a given input set $I, V$ denotes the linear subspace $V=\left\{x \in \mathbf{F}_{2}^{n}: x_{i}=\right.$ $0, i \in I\}$. Then, the sieving probability of $(I, J)$ can be expressed in terms of the sizes of all Range $\left(S_{J}\right)_{\mid u+V}$ when $u$ varies (see Prop 2 in [9]). Most notably, we deduce:

Corollary 1. The sieving probability of $(I, J)$ satisfies $\pi_{I, J} \geq 2^{-p}$, with equality if and only if $S_{J}$ does not depend on its inputs at positions in $\{1, \ldots, n\} \backslash I$.

Link with the branch number of $S$. We associate to $S$ the (nonlinear) code $\mathcal{C}_{S}$ of length $\left(n+n^{\prime}\right)$ and of size $2^{n}$ defined by $\mathcal{C}_{S}=\left\{(x, S(x)), x \in \mathbf{F}_{2}^{n}\right\}$. The minimum distance of $\mathcal{C}_{S}$ is the lowest value of $w t(x+y)+w t(S(x)+S(y))$ for distinct $x, y$. It corresponds to the branch number of $S$. Obviously, when $m+p>n$, the sieving probability of any $(I, J)$ of size $(m, p)$ is at most $2^{n-(m+p)}$ (see Prop 1). Now, the following proposition shows that this upper bound is tight when $(m+p)$ exceeds some bound depending on the branch number of $S$.

Proposition 2. Let $m$ and $p$ be two integers with $m+p \geq n$. Then, all $(m, p)$ sieves have probability $2^{n-(m+p)}$ if and only if $m+p>n+n^{\prime}-d_{\min }$ where $d_{\min }$ is the branch number of $S$ (i.e., the minimal distance of $\mathcal{C}_{S}$ ).

For instance, the branch number of the $4 \times 4$ PRESENT sbox is equal to 3 . It follows that any $(m, p)$ sieve with $m+p \geq 6$ has probability $2^{n-(m+p)}$.

Lower Bound on the Minimal Value of $(m+p)$. Even if the code $\mathcal{C}_{S}$ is a nonlinear code, its dual distance can be defined as follows (if $\mathcal{C}_{S}$ is linear, this definition coincides with the minimum distance of the dual code $\mathcal{C}_{S}^{\perp}$ ).

Definition 2. Let $\mathcal{C}$ be a code of length $N$ and size $M$ over $\mathbf{F}_{q}$ and $A=$ $\left(A_{0}, \ldots, A_{N}\right)$ be its distance distribution, i.e., $A_{i}=\frac{1}{M} \#\{(x, y) \in \mathcal{C} \times \mathcal{C}$ : $\left.d_{H}(x, y)=i\right\}$.

Let $A^{\prime}=\left(A_{0}^{\prime}, \ldots, A_{N}^{\prime}\right)$ be the image of $A$ under the MacWilliams transform, $A^{\prime}(X, Y)=A(X+(q-1) Y, X-Y)$ where $A(X, Y)=\sum_{i=0}^{N} A_{i} X^{N-i} Y^{i}$ and $A^{\prime}(X, Y)=\sum_{i=0}^{N} A_{i}^{\prime} X^{N-i} Y^{i}$. The dual distance of $\mathcal{C}$ is the smallest nonzero index $i$ such that $A_{i}^{\prime} \neq 0$.

The dual distance of $\mathcal{C}_{S}$ is a lower bound on the lowest $(m+p)$ for which an $(m, p)$-sieve exists.

Theorem 1. Let $d^{\perp}$ be the dual distance of the code $\mathcal{C}_{S}$. Then, for any $(m, p)$ such that $m+p<d^{\perp}$, there is no $(m, p)$-sieve for $S$. Moreover, there exists no $(m, p)$-sieve for $S$ with $m+p \leq n$ if and only if $\mathcal{C}_{S}$ is an MDS code, which cannot occur if $S$ is defined over $\mathbf{F}_{2}$.

In some scenarios, $S$ is defined over a larger alphabet, and $I$ and $J$ may be defined as two sets of byte (or nibble) positions. Then, the previous theorem proves that, if the corresponding code $\mathcal{C}_{S}$ is an MDS code, there is no $(m, p)$ sieve for $m+p \leq n$, and we deduce also from Proposition 2 that all $(m, p)$-sieve with $m+p>n$ have probability $2^{n-(m+p)}$. 


\subsection{Sieving Probability for Some Particular Values of $(m, p)$}

$(\boldsymbol{m}, \mathbf{1})$-Sieves and Nonlinearity. When $p=1$, a pair $(I,\{j\})$ of size $(m, 1)$ is a sieve if and only if $S_{j}$ is constant on some coset $u+V$. Therefore, if $(I,\{j\})$ is a sieve, then $S_{j}$ is $(n-m)$-normal, i.e. constant on an affine subspace of dimension $(n-m)$. In particular, it can be approximated by an affine function with a probability at least $\frac{1}{2}\left(1+2^{-m}\right)$ [11. It follows that, if $S$ provides the best resistance to linear cryptanalysis for even $n$, then it has no sieve $(I,\{j\})$ with $|I|<\frac{n}{2}-1$. As an example, the AES Sbox does not have any $(2,1)$-sieve.

$(\boldsymbol{n}-\mathbf{1}, \boldsymbol{p})$-Sieves. When $m=n-1$, the sieving probability can be easily determined by the difference table of $S$.

Proposition 3. Let $I=\{1, \ldots, n\} \backslash\{\ell\}$ and let $J \subset\left\{1, \ldots, n^{\prime}\right\}$ with $|J|=p$. Then,

$$
\pi_{I, J}=2^{-(p-1)}-2^{-(p+n)} \sum_{\beta \in \mathbf{F}_{2}^{n^{\prime}-p}} \delta\left(e_{\ell},\left(0_{J}, \beta\right)\right),
$$

where $\delta(a, b)=\left|\left\{x \in \mathbf{F}_{2}^{n}: S(x+a)+S(x)=b\right\}\right|$ is the element of index $(a, b)$ in the difference table of $S$, and $e_{\ell}$ is the input vector with a 1 at position $\ell$. Thus, $(I,\{j\})$ is a sieve except if $S_{j}$ is linear in $x_{\ell}$.

Since the branch number of the PRESENT sbox is 3, Prop. 2 implies that $(m, p)$ sieves with $m+p=5$ exist for this sbox. Indeed, by considering its difference table, we get that all $(I, J)$ of size $(3,2)$ correspond to a sieving probability $\pi_{I, J} \in\left\{\frac{1}{2}, \frac{1}{2}-\frac{1}{32}, \frac{1}{2}-\frac{1}{16}\right\}$. For instance, the sieve used in the attack in [9], $I=\{0,1,2\}$ and $J=\{0,1\}$ has probability $\frac{1}{2}$. We also derive from Prop. 3 the exact sieving probability involved in the attack on the DES in [9].

\section{Conclusions}

The main contributions of this paper are a generic improvement of MITM attacks, the sieve-in-the-middle technique, which allows to attack more rounds, and an improved biclique construction which avoids the need of additional data. These two methods have been applied to PRESENT, DES, AES and PRINCE. Moreover, some general results on the sieving probability of an sbox are given, which allow to theoretically estimate the complexity of the attack.

A future possible line of work is to investigate some possible combinations with other existing MITM improvements: with the guess of intermediate state bits [12, or with the all-subkeys approach [15]. A promising direction would be to try to make a first selection within each of the two lists before the merging step, by keeping only the input values (resp. output values) which have the lowest probability of corresponding to a valid transition. This introduces some nondetection probability, since some correct candidates would be discarded, but the sieving would be improved. Such an approach does not seem easy, but it would surely be a big step forward for further improving MITM attacks. 
Acknowledgements. We thank Dmitry Khovratovich for his valuable comments, and all CryptoExperts members for their kindness and hospitality.

\section{References}

1. Abed, F., List, E., Lucks, S.: On the Security of the Core of PRINCE Against Biclique and Differential Cryptanalysis. Cryptology ePrint Archive, Report 2012/712 (2012), http://eprint.iacr.org/2012/712

2. Aoki, K., Sasaki, Y.: Preimage Attacks on One-Block MD4, 63-Step MD5 and More. In: Avanzi, R.M., Keliher, L., Sica, F. (eds.) SAC 2008. LNCS, vol. 5381, pp. 103-119. Springer, Heidelberg (2009)

3. Aoki, K., Sasaki, Y.: Meet-in-the-Middle Preimage Attacks Against Reduced SHA0 and SHA-1. In: Halevi, S. (ed.) CRYPTO 2009. LNCS, vol. 5677, pp. 70-89. Springer, Heidelberg (2009)

4. Bogdanov, A., Khovratovich, D., Rechberger, C.: Biclique Cryptanalysis of the Full AES. In: Lee, D.H., Wang, X. (eds.) ASIACRYPT 2011. LNCS, vol. 7073, pp. 344-371. Springer, Heidelberg (2011)

5. Bogdanov, A., Rechberger, C.: A 3-Subset Meet-in-the-Middle Attack: Cryptanalysis of the Lightweight Block Cipher KTANTAN. In: Biryukov, A., Gong, G., Stinson, D.R. (eds.) SAC 2010. LNCS, vol. 6544, pp. 229-240. Springer, Heidelberg (2011)

6. Borghoff, J., Canteaut, A., Güneysu, T., Kavun, E.B., Knezevic, M., Knudsen, L.R., Leander, G., Nikov, V., Paar, C., Rechberger, C., Rombouts, P., Thomsen, S.S., Yalçın, T.: PRINCE - A Low-Latency Block Cipher for Pervasive Computing Applications. In: Wang, X., Sako, K. (eds.) ASIACRYPT 2012. LNCS, vol. 7658, pp. 208-225. Springer, Heidelberg (2012)

7. Bouillaguet, C., Derbez, P., Fouque, P.-A.: Automatic Search of Attacks on RoundReduced AES and Applications. In: Rogaway, P. (ed.) CRYPTO 2011. LNCS, vol. 6841, pp. 169-187. Springer, Heidelberg (2011)

8. Brumley, B.B., Hakala, R.M., Nyberg, K., Sovio, S.: Consecutive S-box Lookups: A Timing Attack on SNOW 3G. In: Soriano, M., Qing, S., López, J. (eds.) ICICS 2010. LNCS, vol. 6476, pp. 171-185. Springer, Heidelberg (2010)

9. Canteaut, A., Naya-Plasencia, M., Vayssière, B.: Sieve-in-the-Middle: Improved MITM Attacks (Full Version). Cryptology ePrint Archive, Report 2013/324 (2013), http://eprint.iacr.org/2013/324

10. Dinur, I., Dunkelman, O., Keller, N., Shamir, A.: Efficient Dissection of Composite Problems, with Applications to Cryptanalysis, Knapsacks, and Combinatorial Search Problems. In: Safavi-Naini, R. (ed.) CRYPTO 2012. LNCS, vol. 7417, pp. 719-740. Springer, Heidelberg (2012)

11. Dobbertin, H.: Construction of Bent Functions and Balanced Boolean Functions with High Nonlinearity. In: Preneel, B. (ed.) FSE 1994. LNCS, vol. 1008, pp. 61-74. Springer, Heidelberg (1995)

12. Dunkelman, O., Sekar, G., Preneel, B.: Improved Meet-in-the-Middle Attacks on Reduced-Round DES. In: Srinathan, K., Rangan, C.P., Yung, M. (eds.) INDOCRYPT 2007. LNCS, vol. 4859, pp. 86-100. Springer, Heidelberg (2007)

13. Guo, J., Ling, S., Rechberger, C., Wang, H.: Advanced Meet-in-the-Middle Preimage Attacks: First Results on Full Tiger, and Improved Results on MD4 and SHA-2. In: Abe, M. (ed.) ASIACRYPT 2010. LNCS, vol. 6477, pp. 56-75. Springer, Heidelberg (2010) 
14. Isobe, T.: A Single-Key Attack on the Full GOST Block Cipher. In: Joux, A. (ed.) FSE 2011. LNCS, vol. 6733, pp. 290-305. Springer, Heidelberg (2011)

15. Isobe, T., Shibutani, K.: All Subkeys Recovery Attack on Block Ciphers: Extending Meet-in-the-Middle Approach. In: Knudsen, L.R., Wu, H. (eds.) SAC 2012. LNCS, vol. 7707, pp. 202-221. Springer, Heidelberg (2013)

16. Jean, J., Nikolic, I., Peyrin, T., Wang, L., Wu, S.: Security Analysis of PRINCE. In: FSE 2013. LNCS. Springer (to appear, 2013)

17. Khovratovich, D., Naya-Plasencia, M., Röck, A., Schläffer, M.: Cryptanalysis of Luffa v2 components. In: Biryukov, A., Gong, G., Stinson, D.R. (eds.) SAC 2010. LNCS, vol. 6544, pp. 388-409. Springer, Heidelberg (2011)

18. Khovratovich, D., Rechberger, C., Savelieva, A.: Bicliques for Preimages: Attacks on Skein-512 and the SHA-2 Family. In: Canteaut, A. (ed.) FSE 2012. LNCS, vol. 7549, pp. 244-263. Springer, Heidelberg (2012)

19. Naya-Plasencia, M.: How to Improve Rebound Attacks. In: Rogaway, P. (ed.) CRYPTO 2011. LNCS, vol. 6841, pp. 188-205. Springer, Heidelberg (2011)

20. Sasaki, Y.: Meet-in-the-Middle Preimage Attacks on AES Hashing Modes and an Application to Whirlpool. IEICE Transactions 96-A(1), 121-130 (2013)

21. Soleimany, H., Blondeau, C., Yu, X., Wu, W., Nyberg, K., Zhang, H., Zhang, L., Wang, Y.: Reflection Cryptanalysis of PRINCE-like Ciphers. In: FSE 2013. LNCS. Springer (to appear, 2013) 